\title{
Magnetic field driven transition between valence bond solid and antiferromagnetic order in a distorted triangular lattice
}

\author{
Yasuhiro Shimizu $\odot,{ }^{1}$ Mitsuhiko Maesato $\odot,{ }^{2}$ Makoto Yoshida, ${ }^{3}$ Masashi Takigawa, ${ }^{3}$ Masayuki Itoh, ${ }^{1}$ Akihiro Otsuka, ${ }^{2,4}$ \\ Hideki Yamochi ${ }^{\circ},{ }^{2,4}$ Yukihiro Yoshida ${ }^{2},{ }^{2,5}$ Genta Kawaguchi, ${ }^{2}$ David Graf, ${ }^{6}$ and Gunzi Saito ${ }^{5,7}$ \\ ${ }^{1}$ Department of Physics, Nagoya University, Chikusa, Nagoya 464-8602, Japan \\ ${ }^{2}$ Division of Chemistry, Graduate School of Science, Kyoto University, Sakyo-ku, Kyoto 606-8502, Japan \\ ${ }^{3}$ Institute for Solid State Physics, University of Tokyo, Kashiwa, Chiba 277-8581, Japan \\ ${ }^{4}$ Research Center for Low Temperature and Materials Sciences, Kyoto University, Sakyo-ku, Kyoto 606-8501, Japan \\ ${ }^{5}$ Faculty of Agriculture, Meijo University, Tempaku-ku, Nagoya 468-8502, Japan \\ ${ }^{6}$ National High Magnetic Field Laboratory, Florida State University, Tallahassee, Florida 32310, USA \\ ${ }^{7}$ Toyota Physical and Chemical Research Institute, Nagakute, Aichi 480-1192, Japan
}

(Received 12 July 2020; revised 9 September 2020; accepted 28 April 2021; published 26 May 2021)

\begin{abstract}
A molecular Mott insulator $\kappa-(\mathrm{ET})_{2} \mathrm{~B}(\mathrm{CN})_{4}[\mathrm{ET}=$ bis(ethylenedithio)tetrathiafulvalene $]$ with a distorted triangular lattice exhibits a quantum disordered state with gapped spin excitation in the ground state. ${ }^{13} \mathrm{C}$ nuclear magnetic resonance, magnetization, and magnetic torque measurements reveal that magnetic field suppresses valence bond order and induces long-range magnetic order above a critical field $\sim 8 \mathrm{~T}$. The nuclear spin-lattice relaxation rate $1 / T_{1}$ shows persistent evolution of antiferromagnetic correlation above the transition temperature, highlighting a quantum spin liquid state with fractional excitations. The field-induced transition as observed in the spin-Peierls phase suggests that the valence bond order transition is driven through renormalized one-dimensionality and spin-lattice coupling.
\end{abstract}

DOI: 10.1103/PhysRevResearch.3.023145

\section{INTRODUCTION}

Quantum magnets featured by macroscopic quantum entanglements host exotic quasiparticles and topological order [1-3]. In frustrated spin systems, a valence bond order state with translational symmetry breaking competes with the magnetic order and quantum spin liquid states [4-7]. The phase transition between magnetic order and valence bond crystal phases may occur through continuous quantum phase transition $[3,8]$, instead of the first-order transition that separates the two phases having competing order parameters in a LandauGinzburg-Wilson paradigm. The realization of deconfined quantum criticality with fractional excitation has been a fundamental issue of many-body quantum physics [4,9-11].

Under an intense magnetic field, the valence bond order state is destabilized and exhibits a transition into long-rangeorder states $[12,13]$. The quasiparticle in the spin-dimer state is a spin $S=1$ triplon localized on the lattice site. The fieldinduced transition involving long-range order of transverse magnetization is regarded as Bose-Einstein condensation of triplons, as observed in several quantum magnets [12-20]. On one-dimensional (1D) systems, the spin-Peierls transition occurs through spin-lattice coupling and is suppressed by

Published by the American Physical Society under the terms of the Creative Commons Attribution 4.0 International license. Further distribution of this work must maintain attribution to the author(s) and the published article's title, journal citation, and DOI. forming a soliton lattice under high magnetic field [21-24]. By contrast, the spin-singlet formation in two-dimensional (2D) frustrated spin systems can be highly degenerate and involve fractionalized quasiparticles [9].

Molecular Mott insulators with a triangular lattice such as $\kappa-(\mathrm{ET})_{2} X$ and $X\left[\mathrm{Pd}(\mathrm{dmit})_{2}\right]_{2} \quad(X$ denotes counterions $)$ may provide the candidate showing the deconfined criticality. Depending on the strength and anisotropy of intermolecular interactions, a series of materials exhibit fertile ground states including quantum spin liquid [25-30], antiferromagnetic order [31,32], and valence bond order [33,34]. Some of them display superconductivity under pressure [35,36]. Here we focus on a Mott insulator $\kappa-(\mathrm{ET})_{2} \mathrm{~B}(\mathrm{CN})_{4}$ having an anisotropic triangular lattice [33]. The anisotropy of exchange interactions is evaluated as $J^{\prime} / J=2\left(J=118 \mathrm{~K}, J^{\prime}=236 \mathrm{~K}\right)$ from the spin susceptibility [37], where $J$ (or transfer integral $t$ ) and $J^{\prime}\left(t^{\prime}\right)$ form a square lattice and a chain, respectively [Fig. 1(a)]. The anisotropy increases up to $J^{\prime} / J \sim 3$, as temperature $(T)$ decreases. It agrees with the calculated transfer anisotropy $t^{\prime} / t=1.4$ or $\left(t^{\prime} / t\right)^{2}=2$ [33] and the optical measurement [38]. Under pressure up to $2.5 \mathrm{GPa}$, the system remains Mott-insulating. Thus the material is located far from the Mott transition at ambient pressure and provides an ideal Heisenberg antiferromagnet with an anisotropic triangular lattice.

The spin susceptibility of $\kappa-(\mathrm{ET})_{2} \mathrm{~B}(\mathrm{CN})_{4}$ exponentially decreases below $T_{c} \sim 5 \mathrm{~K}$ without showing an indication of long-range magnetic order in the ${ }^{1} \mathrm{H}$ NMR spectrum [33]. Thus the ground state likely accompanies the valence bond order with translational symmetry breaking or the spin-Peierls 
(a)

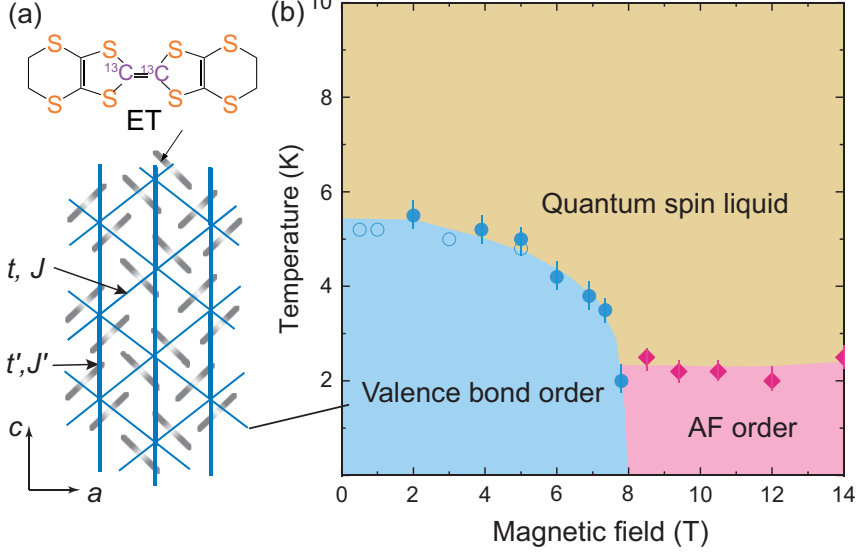

FIG. 1. (a) Anisotropic triangular lattice of molecular dimers in $\kappa$ - $(\mathrm{ET})_{2} \mathrm{~B}(\mathrm{CN})_{4}$ with two kinds of transfers $t$ and $t^{\prime}$ above $T_{c}$. Spin-1/2 on each ET dimer is paired to form a valence bond crystal below $T_{c}=5 \mathrm{~K}$. (b) Magnetic phase diagram based on NMR (closed symbols) and spin susceptibility $\chi$ (open symbols). Circles and diamonds with the uncertainty denote the valence bond order and antiferromagnetic transition temperatures, respectively, which are obtained from the temperature dependence of nuclear spin-lattice relaxation rate $T_{1}^{-1}$.

transition, whereas the crystal structure has not been solved below $T_{c}$. The ${ }^{1} \mathrm{H}$ NMR nuclear-lattice relaxation rate $T_{1}^{-1}$ exhibits weak $T$ dependence above $T_{c}$, consistent with the behavior of the low-dimensional quantum antiferromagnet in the quantum critical region [39]. Considering the low $T_{c}$, an application of magnetic field is expected to induce quantum phase transition, which gives an insight into the origin of the valence bond order and the quasiparticle property.

In this article, we investigate the field-driven transition from the valence bond order phase in $\kappa-(\mathrm{ET})_{2} \mathrm{~B}(\mathrm{CN})_{4}$. The local field distribution and spin excitation are probed by ${ }^{13} \mathrm{C}$ NMR spectroscopy down to low temperatures under magnetic field up to $14 \mathrm{~T}$. Together with the magnetization and torque measurements, we uncover the ground state and low-energy properties across the field-driven transition. The magnetic field $(H)$ versus $T$ phase diagram based on the experimental results [Fig. 1(b)] is compared with the quantum spin liquid and spin-Peierls systems.

\section{EXPERIMENT}

Single crystals of $\kappa-(\mathrm{ET})_{2} \mathrm{~B}(\mathrm{CN})_{4}$ were grown by an electro-oxidation method [33]. The magnetization was measured with a superconducting quantum interference device under $0.5-7 \mathrm{~T}$. The high-field magnetic torque for a single crystal was measured using a piezoresistive cantilever at the National High Magnetic Field Laboratory in Tallahassee, FL. The magnetic field direction was tilted by $16^{\circ}$ from the ac plane. For NMR experiments, the $90 \%{ }^{13} \mathrm{C}$ isotope was selectively enriched to the central double-bonded carbon sites of ET [Fig. 1(a)] [40]. NMR measurements were conducted on a single crystal under the external magnetic field oriented along the $a$ axis $\left(<1^{\circ}\right)$ of the conducting plane by utilizing a two-axis goniometer. The frequency-swept NMR spectrum was obtained from the spin echo measurement with the pulse
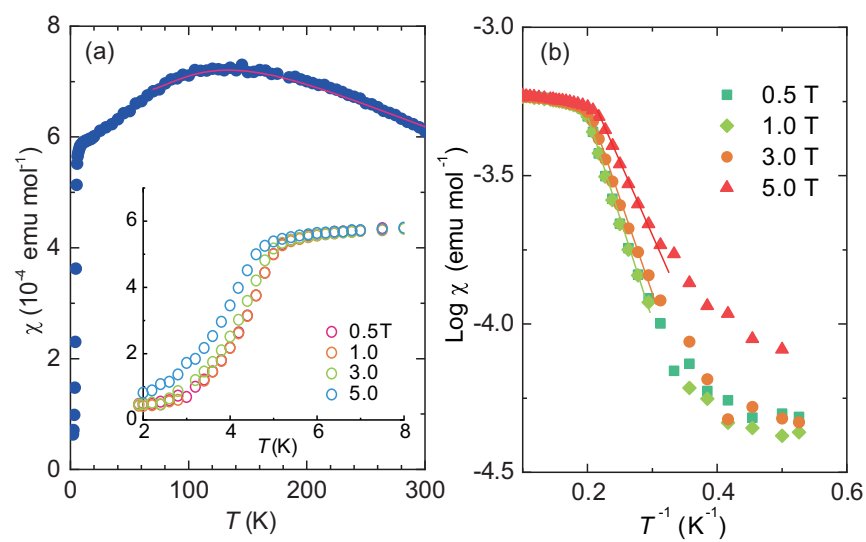

FIG. 2. (a) Spin susceptibility $\chi$ for a polycrystalline sample of $\kappa-(\mathrm{ET})_{2} \mathrm{~B}(\mathrm{CN})_{4}$ at $1 \mathrm{~T}$ [33] after subtracting the core diamagnetic susceptibility $\left(\chi_{\text {dia }}=-4.52 \times 10^{-4} \mathrm{emu} \mathrm{mol}^{-1}\right)$. The red curve is a fitting result by a high-temperature series expansion [37]. Inset: Temperature dependence of $\chi$ at low temperatures under the magnetic field of $0.5,1,3$, and $5 \mathrm{~T}$. (b) Inverse temperature dependence of $\chi$ for the evaluation of spin gap with the exponential function $\chi \sim \exp (-\Delta / T)$.

width $t_{\pi / 2}=0.5 \mu \mathrm{s}$ and $t_{\pi}=1 \mu \mathrm{s}$, and the sum was taken by $0.2 \mathrm{MHz}$ steps covering the whole spectrum at low temperatures [Fig. 5(b)]. The nuclear magnetization recovery after the saturation followed a single exponential function above $5 \mathrm{~K}$ and becomes a stretched exponential function $M(t) / M_{0}=$ $1-\exp \left[-\left(t / T_{1}\right)^{\beta}\right]$ with the exponent $\beta=0.3-1$ below $5 \mathrm{~K}$, which is nearly independent of $H$.

\section{EXPERIMENTAL RESULTS}

\section{A. Spin susceptibility and Knight shift}

The spin susceptibility $\chi$ of $\kappa-(\mathrm{ET})_{2} \mathrm{~B}(\mathrm{CN})_{4}$ exhibits a broad maximum around $130 \mathrm{~K}$ [33], characteristic of the low-dimensional antiferromagnet, as shown in Fig. 2(a). The $T$ dependence is fitted into a high- $T$ series expansion of the Heisenberg model with an anisotropic triangular lattice $(J=$ $118 \mathrm{~K}, J^{\prime}=236 \mathrm{~K}$ ) [37]. Below $T_{c}=5 \mathrm{~K}, \chi$ measured at $1 \mathrm{~T}$ decreases continuously, indicating the second-order phase transition. The spin gap was evaluated as $\Delta=27 \mathrm{~K}$ for the singlet-triplet model [33] at $1 \mathrm{~T}$. Here we employ the simple exponential function [Fig. 2(b)], yielding $\Delta=16,14.8$, and $11.1 \mathrm{~K}$ under $H=1,3$, and $5 \mathrm{~T}$, respectively. The negligible Curie tail increase in $\chi$ at low temperatures verifies the high crystal quality.

The ${ }^{13} \mathrm{C}$ NMR spectrum provides the local spin susceptibility and the local field distribution at nuclear sites through the hyperfine interaction. In $\kappa-(\mathrm{ET})_{2} \mathrm{~B}(\mathrm{CN})_{4}$, each ET molecule has two ${ }^{13} \mathrm{C}$ sites, all of which in the unit cell (orthorhombic Pnma) are equivalent [Fig. 1(a)]. Therefore, the NMR spectrum in Fig. 3(a) consists of two doublets due to dipolecoupled two ${ }^{13} \mathrm{C}$ spins [41]. Upon cooling, the spectrum gradually broadens toward $T_{c}$, coinciding with the intensity reduction due to fluctuations and the inhomogeneous field due to the generation of paramagnetic domains around the structural transition. Below $T_{c}$, the spectrum narrows, and only the doublet splitting remains. The splitting frequency is about 

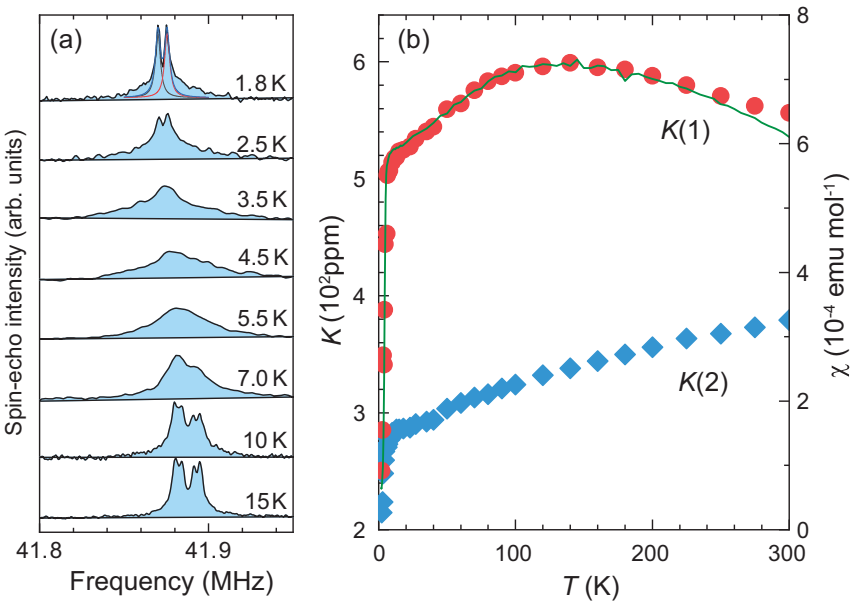

FIG. 3. (a) ${ }^{13} \mathrm{C}$ NMR spectra at $H=3.9 \mathrm{~T}$ along the $a$ axis in $\kappa-(\mathrm{ET})_{2} \mathrm{~B}(\mathrm{CN})_{4}$. Lorentzian fitting curves are shown at the lowest temperature. (b) Knight shifts, $K(1)$ and $K(2)$, for two carbon sites, $\mathrm{C}(1)$ and $\mathrm{C}(2)$, which are measured from the reference (tetramethylsilane). A green curve denotes the spin susceptibility $\chi$ at $1.0 \mathrm{~T}$ (the right-hand axis) [33].

$3 / 2$ times larger than that observed at high temperatures, which represents a crossover from the unlike-spin to like-spin coupling, as the spin susceptibility vanishes below $T_{c}$ [41]. Since ${ }^{13} \mathrm{C}$ spins $(I=1 / 2)$ do not have electric quadrupole interaction, structural fluctuation is observed through a small nuclear dipole coupling and paramagnetic domains.

The residual broad tails of the spectrum at low temperatures can be attributed to staggered moments nucleated around the domains through the Dzyaloshinskii-Moriya (DM) interaction [32]. The sharp central line means that the majority of spins remain nonmagnetic.

The Knight shift $K$ proportional to the local spin susceptibility $\chi_{\text {loc }}$ is defined for two carbon sites on each ET molecule, termed $K(1)$ and $K(2)$. As shown in Fig. 3(b), $K(1)$ exhibits a broad maximum around $130 \mathrm{~K}$ as observed in $\chi$, reflecting the short-range antiferromagnetic correlation. In contrast, $K(2)$ monotonously decreases upon cooling. The site dependence implies that the hyperfine coupling and the chemical shift vary with $T$ due to the anisotropic lattice contraction [33].

At low temperatures below $50 \mathrm{~K}$, the Knight shifts scale well to $\chi$. The linearity in the $K-\chi$ plot for $10-50 \mathrm{~K}$ yields the hyperfine coupling constant $H_{\mathrm{hf}}$, as shown in Fig. 4. In the present field direction $(H \| a)$, we obtained $H_{\mathrm{hf}}=$ $0.34(2) T / \mu_{\mathrm{B}}$ for $\mathrm{C}(1)$ and $0.18(4) \mathrm{T} / \mu_{\mathrm{B}}$ for $\mathrm{C}(2)$. The difference reflects the asymmetric spin density distribution due to the molecular dimerization [32]. Here $H_{\mathrm{hf}}$ is composed of the Fermi contact and dipolar interaction terms. The anisotropic dipole term may depend on temperature when the principal axis governed by the strength of the dimerization changes upon cooling. Similar behavior has been observed in the other members of $\kappa-(\mathrm{ET})_{2} X[32,42]$.

\section{B. Magnetic field driven transition}

The field sensitivity of the spin gap implies that a quantum phase transition occurs at the critical field $H_{c}$ where $\Delta$ goes to zero or competes with the other order parameter.

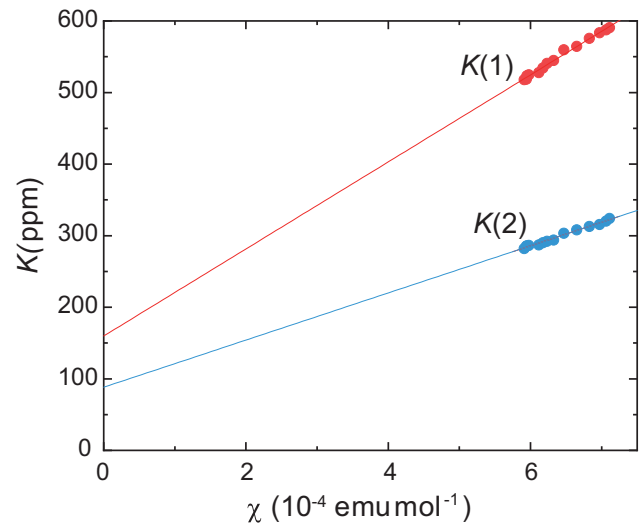

FIG. $4 .{ }^{13} \mathrm{C}$ Knight shifts plotted against $\chi$ for $10-50 \mathrm{~K}$. The linearity yields the hyperfine coupling constant for two ${ }^{13} \mathrm{C}$ sites. The $y$-intercept gives the chemical shift.

For investigating the field-driven transition, we measured the magnetic torque $\tau$ responsive to the transverse magnetization, $\tau / H \sim M_{T}$, as shown in Fig. 5. In a paramagnetic state above $T_{c}, \tau / H$ is governed by the $g$-value anisotropy and increases with the external field $H$. The slop is nearly independent of temperature at high fields.

Below $4.5 \mathrm{~K}, \tau / H$ becomes nonlinear to $H . \tau / H$ behaves constantly at low fields, reflecting $\chi \simeq 0$ with a negligible Curie impurity component. Then $\tau / H$ starts to increase around $8 \mathrm{~T}$, which clearly indicates a magnetic transition from the nonmagnetic state into the magnetically ordered state or the paramagnetic spin liquid state. Below $2.5 \mathrm{~K}$, a maximum appears in $\tau / H$ around 11-12 $\mathrm{T}$. After showing a minimum around 24-28 T, $\tau / H$ linearly increases with $H$

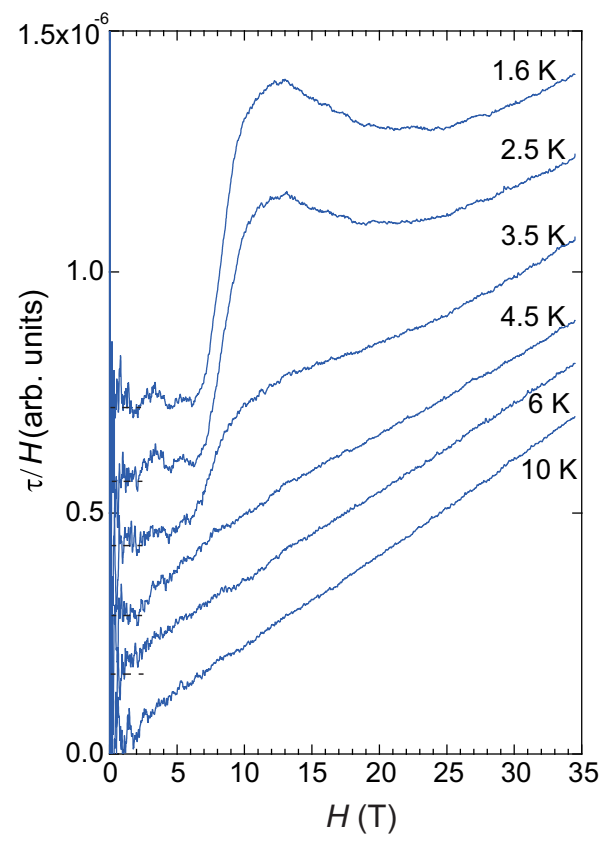

FIG. 5. Magnetic torque $\tau$ divided by magnetic field $H$ at low temperatures. The data at each temperature include a constant offset for clarity. 

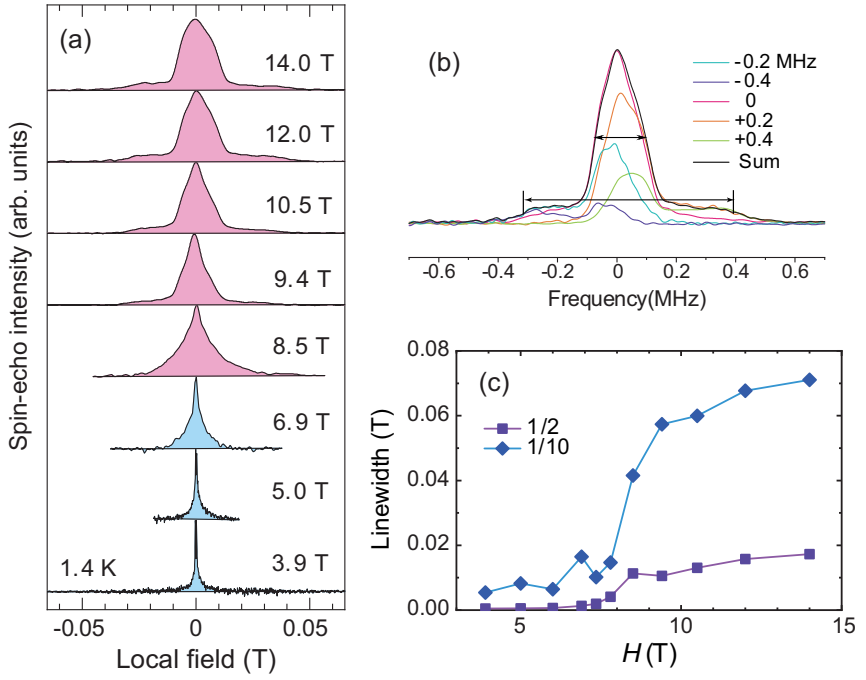

FIG. 6. (a) Magnetic field dependence of the ${ }^{13} \mathrm{C}$ NMR spectrum for $\kappa-(\mathrm{ET})_{2} \mathrm{~B}(\mathrm{CN})_{4}$ at $1.4 \mathrm{~K}$. Frequency $\omega$ was converted to the local field $\Delta B$ using a relation $\Delta B=\Delta \omega / \gamma_{0}\left(\gamma_{0}=10.7054 \mathrm{MHz} / \mathrm{T}\right)$. (b) Definition of the $1 / 2$ and $1 / 10$ linewidths for $T=1.4 \mathrm{~K}$ and $H=$ $12 \mathrm{~T}$, where the spectrum is constructed as a sum of Fourier transformed spin-each intensities taken with a $0.2 \mathrm{MHz}$ step. (c) Magnetic field dependence of the $1 / 2$ and $1 / 10$ linewidths.

without saturation up to $35 \mathrm{~T}$. However, the nature of the strong field-induced phase is unclear at the present stage.

The magnetic field dependence of the ${ }^{13} \mathrm{C}$ NMR spectrum was measured under a magnetic field of 3.9-14 $\mathrm{T}$ at $1.4 \mathrm{~K}$, as displayed in Fig. 6(a). The spectrum gradually spreads even at low fields, indicating the evolution of the local field distribution upon increasing $H$. Above $8.5 \mathrm{~T}$, the spectral broadening becomes prominent at low temperatures. The NMR spectrum eventually changes into a two-step bell shape. The emergence of the huge local field signals long-range magnetic order. Under $H=14 \mathrm{~T}$, the maximum local field reaches $\pm 0.04 \mathrm{~T}$ at the tails of the spectrum.

As shown in Figs. 6(b) and 6(c), the linewidths defined at $1 / 2$ and $1 / 10$ of the maximum intensity evolve above the critical field $H_{c} \sim 8 \mathrm{~T}$ and level off at high fields, consistent with the magnetic torque result. The $1 / 2$ and $1 / 10$ widths measure the evolution of the order parameter at two ${ }^{13} \mathrm{C}$ sites with different $H_{\mathrm{hf}}$. Indeed, the $1 / 2$ width $(0.03 \mathrm{~T})$ and $1 / 10$ width $(0.07 \mathrm{~T})$ approximately scale to $H_{\mathrm{hf}}$ of two ${ }^{13} \mathrm{C}$ sites. The result yields the maximum moment of $\sim 0.1 \mu_{\mathrm{B}}$, which continuously grows above $H_{c}$ and saturates at high fields.

The temperature dependence of NMR spectra above $H_{c}$ is shown in Fig. 7. At $8.5 \mathrm{~T}$, the spectral broadening starts below $10 \mathrm{~K}$ and continues upon cooling, while leaving a narrow line at the central position [Fig. 7(a)]. It suggests the coexistence of the magnetic phase and the valence bond order phase, or the formation of a soliton lattice as observed in the 1D chains [22]. However, the singular edge peak characteristic of solitons was not detected within the experimental uncertainty. At $14 \mathrm{~T}$, the site-dependent broadening becomes clearer above $T_{\mathrm{N}}$. The asymmetric spectral shape in the paramagnetic state becomes nearly symmetric below $2.5 \mathrm{~K}$, which gives evidence for the magnetic order transition.

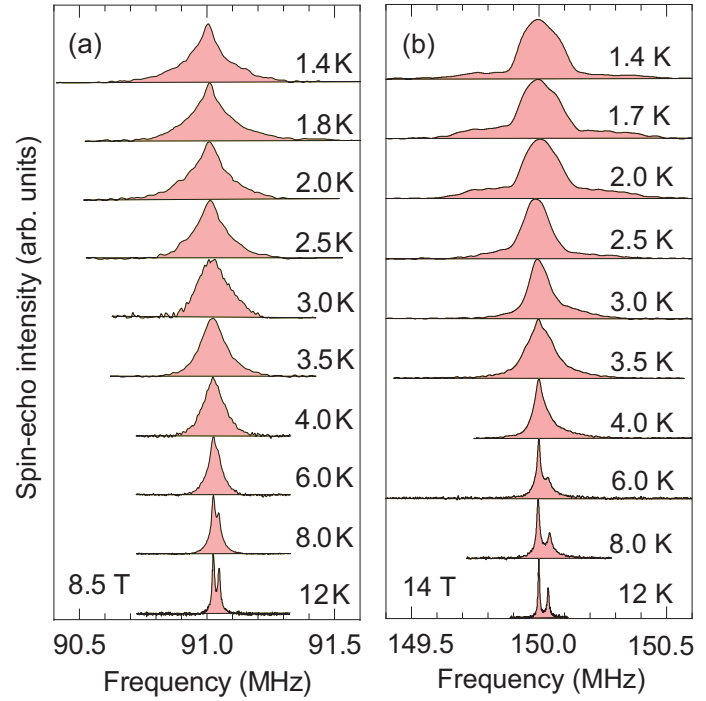

FIG. 7. Temperature dependence of the ${ }^{13} \mathrm{C}$ NMR spectrum for the single crystal of $\kappa-(\mathrm{ET})_{2} \mathrm{~B}(\mathrm{CN})_{4}$ under a magnetic field of (a) 8.5 and (b) $14 \mathrm{~T}$ along the $a$ axis.

In reference to the spin structure of $\kappa-(\mathrm{ET})_{2} \mathrm{Cu}\left[\mathrm{N}(\mathrm{CN})_{2}\right] \mathrm{Cl}$ belonging to the same space group as $\kappa-(\mathrm{ET})_{2} \mathrm{~B}(\mathrm{CN})_{4}$, the collinear magnetic order splits the ${ }^{13} \mathrm{C}$ NMR spectrum under the magnetic field along the easy axis $(H \| c)$ and gives only a positive shift for the perpendicular direction $(H \| a)$ [43]. The observed continuous and symmetric broadening points to an incommensurate magnetic structure such as a spiral order expected for a triangular lattice antiferromagnet $[7,44,45]$. The full determination of the spin structure requires the $H_{\mathrm{hf}}$ tensor and the angular dependence measurements. The sizable moment contraction highlights a crucial role of quantum fluctuations for the system near the disordered state.

\section{Spin dynamics across a field-driven transition}

The spin excitation is investigated by the nuclear spinlattice relaxation rate $T_{1}^{-1}$ across $H_{c}$. As shown in Fig. 8, $T_{1}^{-1}$ is nearly independent of $T$ above $T_{c}$, as expected in lowdimensional quantum antiferromagnets for a $T$ range lower than the exchange coupling $(T<J)$ [39]. The behavior differs from that observed in a triangular lattice system having the spin liquid ground state, as discussed below. $T_{1}^{-1}$ decreases steeply as the spin gap $\Delta$ opens below $T_{c}$ in a low-field range below 8 T. $T_{1}^{-1}$ is enhanced with increasing $H$ as $T_{c}$ and $\Delta$ are suppressed toward $H_{c} \sim 8 \mathrm{~T}$. The obtained field dependence of $T_{c}$ is plotted in Fig. 1(b), consistent with the result of $\chi$.

We evaluated $\Delta$ by fitting $T_{1}^{-1}$ into an exponential function $\sim \exp (-\Delta / T)$ [Fig. 8(b)]. We obtained $\Delta=14.5,12.0$, and $7.2 \mathrm{~K}$ at $H=3.9,5.0$, and $6.9 \mathrm{~T}$, respectively, as plotted in Fig. 8(c). The results are consistent with those obtained from $\chi(T)$ [Fig. 2(b)]. Since $T_{1}^{-1}$ measures the dynamical spin correlation summed over the wave-vector space, the result represents the presence of the spin excitation gap independent of the wave vector. We find that $\Delta$ linearly decreases with increasing $H, \Delta \sim\left(H_{c}-H\right)^{z}$, with $H_{c} \sim 8.2(2) \mathrm{T}$ and the critical exponent $z=1.0(3)$. The $H$ and $T$ dependence of $T_{1}^{-1}$ is also illustrated as a contour plot [Fig. 4(c)]. Approaching 

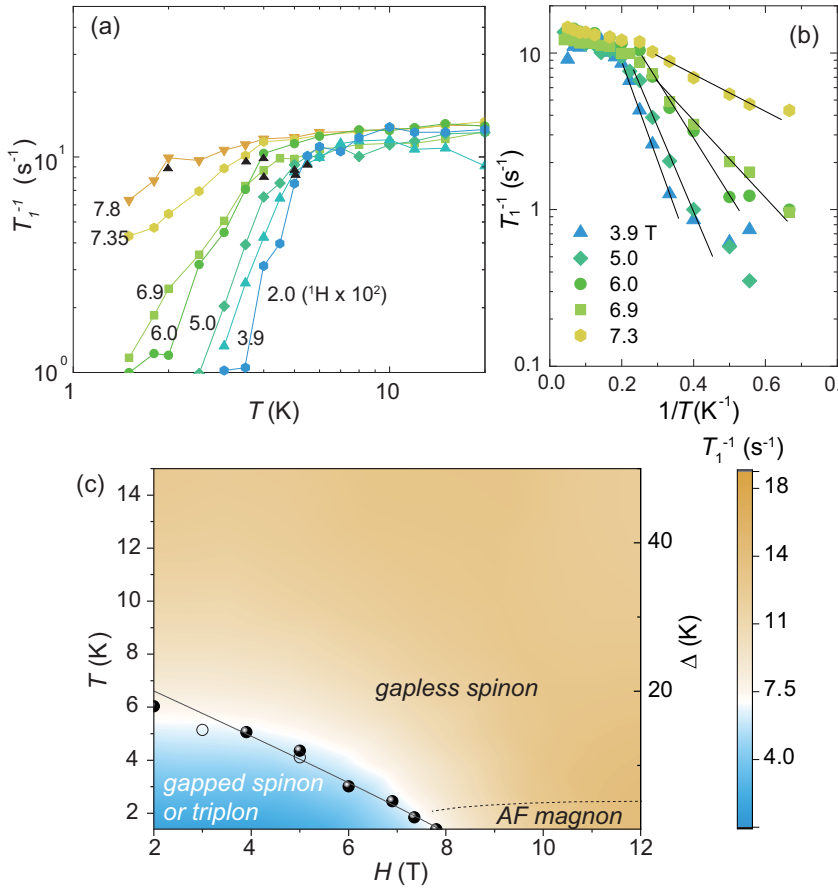

FIG. 8. (a) Temperature dependence of the ${ }^{13} \mathrm{C}$ nuclear spinlattice relaxation rate $T_{1}^{-1}$ at $3.9-7.8 \mathrm{~T}$. ${ }^{1} \mathrm{H}$ NMR $T_{1}^{-1}$ (a long component) at $2.0 \mathrm{~T}$ scales to ${ }^{13} \mathrm{C}$ data by multiplying $10^{2}$ [33]. $T_{c}$ defined by the onset of $T_{1}^{-1}$ drop is marked by arrows. (b) Inverse temperature dependence of $T_{1}^{-1}$ for evaluating the spin gap $\Delta$. (c) Field dependence of $\Delta$ (the right-hand axis) and the contour plot of $T_{1}^{-1}$ obtained from the temperature and field dependence measurement. Solid and open circles are evaluated from $T_{1}^{-1}$ and $\chi$, respectively.

$H_{c}$, the $T$-independent region persists to low temperatures, as expected in the gapless spinon regime.

Above $H_{c}, T_{1}^{-1}$ exhibits a peak structure at $2-2.5 \mathrm{~K}$ due to the slowing down of spin fluctuations, as shown in Fig. 9(a). The peak determines the magnetic order transition temperature $T_{\mathrm{N}}$. It is nearly independent of $H$, as plotted in Fig. 1(b). Below $T_{\mathrm{N}}$, one can expect an increase in the linewidth of
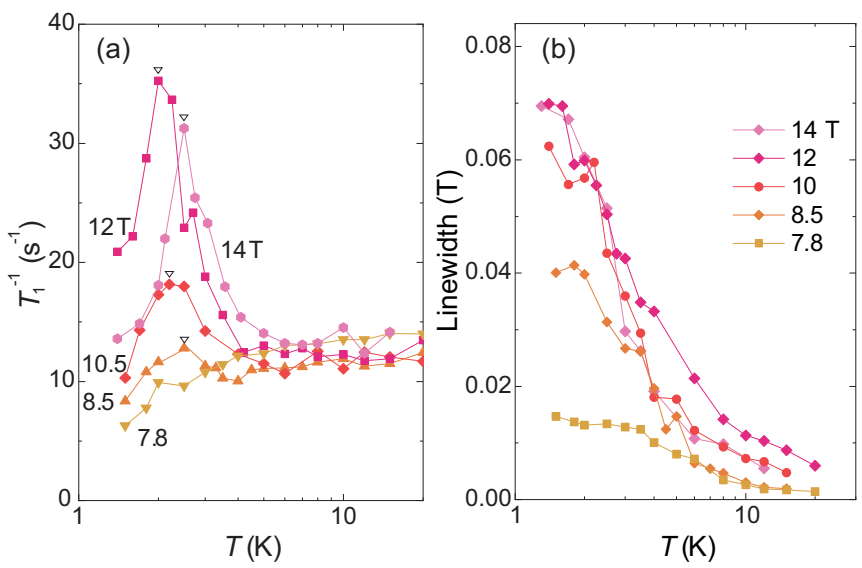

FIG. 9. (a) Temperature dependence of $T_{1}^{-1}$ above $7.8 \mathrm{~T}$. Arrows point to the magnetic order transition at $T_{\mathrm{N}}$. (b) Linewidth of the ${ }^{13} \mathrm{C}$ NMR spectrum, defined by $1 / 10$ of the peak intensity in Fig. 6(b).

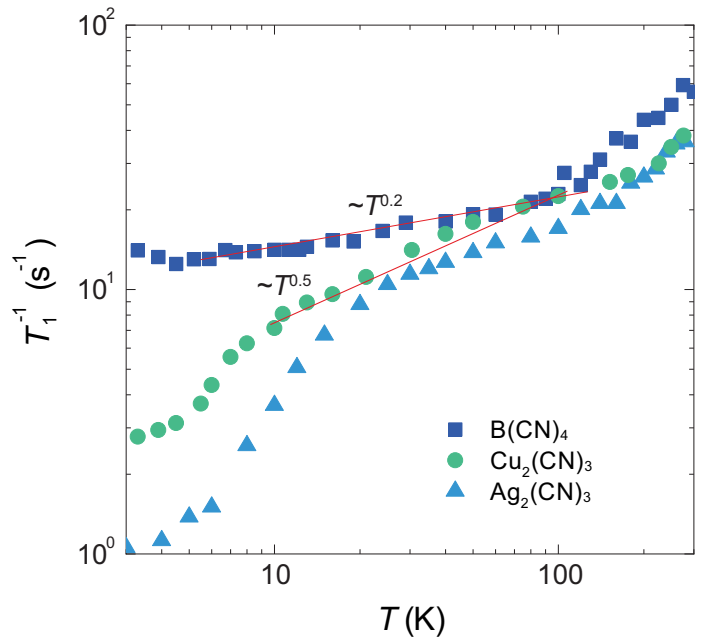

FIG. 10. Temperature dependence of $T_{1}^{-1}$ for ${ }^{13} \mathrm{C}$ NMR in $\kappa$-(ET) $)_{2} X, X=\mathrm{B}(\mathrm{CN})_{4}, \mathrm{Cu}_{2}(\mathrm{CN})_{3}$ [42], and $\mathrm{Ag}_{2}(\mathrm{CN})_{3}$ [32].

the ${ }^{13} \mathrm{C}$ NMR spectrum, which measures the evolution of the order parameter. As shown in Fig. 9(b), the linewidth remains small at $7.8 \mathrm{~T}$ just below $H_{c}$. The spectral broadening starts from $T>T_{\mathrm{N}}$ above $8.5 \mathrm{~T}$ due to fluctuations and inhomogeneity, while the broadening levels off below $T_{\mathrm{N}}$ defined by $T_{1}^{-1}$.

\section{DISCUSSION}

In this section, we focus on the nature of the dynamical spin correlation in the paramagnetic spin liquid phase. We also discuss the origin for the valence bond order transition based on the magnetic phase diagram as well as the possible magnetic phase at high fields.

The series of $\kappa$-(ET) $)_{2} X\left[X=\mathrm{Cu}_{2}(\mathrm{CN})_{3}, \mathrm{Ag}_{2}(\mathrm{CN})_{3}\right]$ have been extensively investigated as frustrated quantum antiferromagnets with a triangular lattice close to the Mott transition $[25,30,32]$, where the anisotropy $t^{\prime} / t$ is close to or smaller than unity. $\kappa-(\mathrm{ET})_{2} \mathrm{~B}(\mathrm{CN})_{4}$ is the rare example of a frustrated triangular lattice with one-dimensional anisotropy $\left(t^{\prime} / t>1\right)$. Thus it is interesting to compare the difference in the dynamical spin correlation between these systems. In the temperature range lower than the antiferromagnetic exchange interaction, the system is regarded as a quantum spin liquid involving low-lying excitations. $T_{1}^{-1}$ measures the dynamical spin susceptibility in the low-energy limit $[9,39]$. On a isotropic triangular lattice such as $\kappa-(\mathrm{ET})_{2} \mathrm{Ag}_{2}(\mathrm{CN})_{3}$ showing a quantum spin liquid state, $T_{1}^{-1}$ follows a power law $\sim T^{\eta}(20<T<$ $200 \mathrm{~K}$ ) with the anomalous exponent $\eta=0.4-0.5$, as shown in Fig. 10 [32,42]. Interestingly, the exponent is close to that expected for deconfined spinon excitations $(\eta=0.6)$ in the frustrated model [9].

In contrast, $T_{1}^{-1}$ for $\kappa$-(ET) ${ }_{2} \mathrm{~B}(\mathrm{CN})_{4}$ follows a power law $\sim T^{\eta}$ with the small exponent $\eta=0.19(1)$ in a wide range $(5<T<100 \mathrm{~K})$, as shown in Fig. 10. Here the strong frustration effect is manifested in the low $T_{c}$ and $T_{\mathrm{N}}$ compared with the exchange coupling. The exponent is rather close to that of the 1D quantum critical antiferromagnet, where $T_{1}^{-1}$ behaves $T$-independent $\left(\sim T^{\eta}, \eta=0\right)$, as the antiferromagnetic 
correlation length $\xi$ diverges with $\xi \sim T^{-z}$ (the dynamical exponent $z=1$ ) at the specific wave vector [39]. The behavior was observed in $\mathrm{Sr}_{2} \mathrm{CuO}_{3}$ [46] and (TMTTF) ${ }_{2} \mathrm{PF}_{6}$ [47].

On the magnetic phase diagram [Fig. 1(b)], the valence bond order phase is suppressed toward the critical field $H_{c} \sim$ $8 \mathrm{~T}$, as observed by magnetic torque and NMR spectrum measurements. It is also consistent with the field dependence of the spin gap $\Delta$ obtained from $T_{1}^{-1}$. The continuous suppression of $\Delta$ implies the quantum phase transition in the frustrated spin system $[3,10,11]$. However, the incommensurate magnetic order phase appears from the finite temperature across $H_{c}$. It implies that the second-order valence bond order transition is terminated by the first-order magnetic transition at low temperatures as anticipated from the mean-field theory $[48,49]$.

The shape of the phase diagram is similar to those of the quasi-1D spin-Peierls system, such as $\mathrm{CuGeO}_{3}$ and $(\mathrm{TMTTF})_{2} \mathrm{PF}_{6}[22,47,50,51]$. It suggests that the valence bond order occurs through the lattice dimerization along the $c$ axis. Indeed, the crystal structure becomes more 1D upon cooling [33]. This tendency is distinct from that observed in the other $\kappa$-(ET) ${ }_{2} X$ members with $t^{\prime} / t<1$ [30]. Thus the renormalized 1D spin correlation can develop in the gapless spin liquid regime above $T_{c}$ [21] and eventually couple the lattice degrees of freedom. Furthermore, the discrete (not polymeric) counterion and the absence of structural disorder allows the spin dimerization along one direction.

Focusing on the magnetically ordered phase at high fields, the magnetic structure would be highly degenerate and nontrivial on the triangular lattice. Theoretical calculations have been reported based on the Hubbard and Heisenberg model as a function of anisotropy of a triangular lattice, $t^{\prime} / t$ or $J^{\prime} / J[7,45,52]$. For $J^{\prime} / J>1$, the magnetic ground state of the Mott insulator can be both quantum spin liquid and antiferromagnetic orders such as collinear and spiral spin structures. Indeed, the isostructure Mott insulator $\kappa$-(ET) ${ }_{2} \mathrm{CF}_{3} \mathrm{SO}_{3}\left(t^{\prime} / t=1.8\right)$ exhibits the magnetically ordered ground state with an incommensurate structure below $T_{\mathrm{N}}=2.5 \mathrm{~K}$ [53]. Therefore, the present system is naturally expected to exhibit long-range magnetic order, as the valence bond crystal is suppressed by the magnetic field.

\section{CONCLUSION}

In conclusion, the field-induced quantum phase transition was observed from the valence bond order to the antiferromagnetic long-range order state by ${ }^{13} \mathrm{C}$ NMR and magnetization measurements in the Mott insulator $\kappa$-(ET) $)_{2} \mathrm{~B}(\mathrm{CN})_{4}$ having a distorted triangular lattice. We found that the spin excitation gap is linearly suppressed by magnetic field toward the critical point. As the valence bond order phase melts, the quantum spin liquid with gapless excitation appears above the critical field. The obtained phase diagram manifests the competing magnetic ground states in the frustrated magnet. The significant role of quantum fluctuations is highlighted as the reduction of the antiferromagnetic moment and the persistent low-lying excitation down to low temperatures.

\section{ACKNOWLEDGMENTS}

This work was financially supported by Grants-in-Aid for Scientific Research (Grants No. JP16H02206, No. JP16H04012, No. JP17H05151, No. JP18H04223, No. JP19H05824, No. JP19H01837, and No. JP20H02709) from Japan Society for the Promotion of Science, and partly supported by the Kyoto University Foundation.
[1] P. W. Anderson, Resonating valence bonds: a new kind of insulator? Mater. Res. Bull. 8, 153 (1973).

[2] X.-G. Wen, Quantum orders and symmetric spin liquids, Phys. Rev. B 65, 165113 (2002).

[3] T. Senthil, A. Vishwanath, L. Balents, S. Sachdev, and M. P. A. Fisher, Deconfined quantum critical points, Science 303, 1490 (2004).

[4] N. Read and S. Sachdev, Large-N Expansion for Frustrated Quantum Antiferromagnets, Phys. Rev. Lett. 66, 1773 (1991).

[5] J. Alicea, O. I. Motrunich, M. Hermele, and M. P. A. Fisher, Criticality in quantum triangular antiferromagnets via fermionized vortices, Phys. Rev. B 72, 064407 (2005).

[6] P. Hauke, Quantum disorder in the spatially completely anisotropic triangular lattice, Phys. Rev. B 87, 014415 (2013).

[7] L. F. Tocchio, C. Gros, R. Valentí, and F. Becca, Onedimensional spin liquid, collinear, and spiral phases from uncoupled chains to the triangular lattice, Phys. Rev. B 89, 235107 (2014).

[8] S. Sachdev, Quantum Phase Transitions, 2nd ed. (Cambridge University Press, London, 2011).

[9] T. Senthil, L. Balents, S. Sachdev, A. Vishwanath, and M. P. A. Fisher, Quantum criticality beyond the Landau-GinzburgWilson paradigm, Phys. Rev. B 70, 144407 (2004).
[10] C.-M. Jian, A. Thomson, A. Rasmussen, Z. Bi, and C. Xu, Deconfined quantum critical point on the triangular lattice, Phys. Rev. B 97, 195115 (2018).

[11] H. D. Scammell and O. P. Sushkov, Violation of the SpinStatistics Theorem and the Bose-Einstein Condensation of Particles With Half-Integer Spin, Phys. Rev. Lett. 114, 055702 (2015).

[12] V. Zapf, M. Jaime, and C. D. Batista, Bose-Einstein condensation in quantum magnets, Rev. Mod. Phys. 86, 563 (2014).

[13] T. Giamarchi, C. Ruegg, and O. Tchernyshyov, Bose-Einstein condensation in magnetic insulators, Nat. Phys. 4, 198 (2008).

[14] K. Shirasawa, N. Kurita, and H. Tanaka, Universality of magnetic-field-induced Bose-Einstein condensation of magnons, Phys. Rev. B 96, 144404 (2017).

[15] F. Yamada, T. Ono, H. Tanaka, G. Misguich, M. Oshikawa, and T. Sakakibara, Magnetic-field induced bose-einstein condensation of magnons and critical behavior in interacting spin dimer system $\mathrm{TlCuCl}_{3}$, J. Phys. Soc. Jpn. 77, 013701 (2008).

[16] O. Vyaselev, M. Takigawa, A. Vasiliev, A. Oosawa, and H. Tanaka, Field-Induced Magnetic Order and Simultaneous Lattice Deformation in $\mathrm{TlCuCl}_{3}$, Phys. Rev. Lett. 92, 207202 (2004). 
[17] V. V. Mazurenko, M. V. Valentyuk, R. Stern, and A. A. Tsirlin, Nonfrustrated Interlayer Order and Its Relevance to the Bose-Einstein Condensation of Magnons in $\mathrm{BaCuSi}_{2} \mathrm{O}_{6}$, Phys. Rev. Lett. 112, 107202 (2014).

[18] S. E. Sebastian, N. Harrison, C. D. Batista, L. Balicas, M. Jaime, P. A. Sharma, N. Kawashima, and I. R. Fisher, Dimensional reduction at a quantum critical point, Nature (London) 441, 617 (2006).

[19] S. Krämer, R. Stern, M. Horvatić, C. Berthier, T. Kimura, and I. R. Fisher, Nuclear magnetic resonance evidence for a strong modulation of the Bose-Einstein condensate in $\mathrm{BaCuSi}_{2} \mathrm{O}_{6}$, Phys. Rev. B 76, 100406(R) (2007).

[20] T. Lancaster, P. A. Goddard, S. J. Blundell, F. R. Foronda, S. Ghannadzadeh, J. S. Möller, P. J. Baker, F. L. Pratt, C. Baines, L. Huang, J. Wosnitza, R. D. McDonald, K. A. Modic, J. Singleton, C. V. Topping, T. A. W. Beale, F. Xiao, J. A. Schlueter, A. M. Barton, R. D. Cabrera et al., Controlling Magnetic Order and Quantum Disorder in Molecule-Based Magnets, Phys. Rev. Lett. 112, 207201 (2014).

[21] Y. Hayashi and M. Ogata, Possibility of gapless spin liquid state by one-dimensionalization, J. Phys. Soc. Jpn. 76, 053705 (2007).

[22] M. Horvatic, Y. Fagot-Revurat, C. Berthier, G. Dhalenne, and A. Revcolevschi, NMR Imaging of the Soliton Lattice Profile in the Spin-Peierls Compound $\mathrm{CuGeO}_{3}$, Phys. Rev. Lett. 83, 420 (1999).

[23] F. Casola, T. Shiroka, A. Feiguin, S. Wang, M. S. Grbić, M. Horvatić, S. Krämer, S. Mukhopadhyay, K. Conder, C. Berthier, H.-R. Ott, H. M. Rønnow, C. Rüegg, and J. Mesot, Field-Induced Quantum Soliton Lattice in a Frustrated Two-Leg Spin-1/2 Ladder, Phys. Rev. Lett. 110, 187201 (2013).

[24] V. Kiryukhin, B. Keimer, and D. E. Moncton, Direct Observation of a Magnetic Field Induced CommensurateIncommensurate Transition in a Spin-Peierls System, Phys. Rev. Lett. 74, 1669 (1995).

[25] Y. Shimizu, K. Miyagawa, K. Kanoda, M. Maesato, and G. Saito, Spin Liquid State in an Organic Mott Insulator With a Triangular Lattice, Phys. Rev. Lett. 91, 107001 (2003).

[26] T. Itou, A. Oyamada, S. Maegawa, and R. Kato, Instability of a quantum spin liquid in an organic triangular-lattice antiferromagnet, Nat. Phys. 6, 673 (2010).

[27] T. Isono, H. Kamo, A. Ueda, K. Takahashi, M. Kimata, H. Tajima, S. Tsuchiya, T. Terashima, S. Uji, and H. Mori, Gapless Quantum Spin Liquid in an Organic Spin-1/2 TriangularLattice $\mathrm{H}_{3}$ (Cat-EDT-TTF $)_{2}$, Phys. Rev. Lett. 112, 177201 (2014).

[28] F. Pratt, P. J. Baker, S. J. Blundell, T. Lancaster, S. OhiraKawamura, C. Baines, Y. Shimizu, K. Kanoda, I. Watanabe, and G. Saito, Magnetic and nonmagnetic phases of a quantum spin liquid, Nature (London) 471, 612 (2011).

[29] T. Hiramatsu, Y. Yoshida, G. Saito, A. Otsuka, H. Yamochi, M. Maesato, Y. Shimizu, H. Ito, Y. Nakamura, H. Kishida, M. Watanabe, and R. Kumai, Design and preparation of a quantum spin liquid candidate $\kappa$-(ET) $)_{2} \mathrm{Ag}_{2}(\mathrm{CN})_{3}$ having a nearby superconductivity, Bull. Chem. Soc. Jpn. 90, 1073 (2017).

[30] K. Kanoda and R. Kato, Mott physics in organic conductors with triangular lattices, Annu. Rev. Condens. Matter Phys. 2, 167 (2011)

[31] K. Miyagawa, K. Kanoda, and A. Kawamoto, NMR studies on two-dimensional molecular conductors and superconductors:
Mott transition in $\kappa$-(BEDT-TTF $)_{2}$ X, Chem. Rev. 104, 5635 (2004).

[32] Y. Shimizu, T. Hiramatsu, M. Maesato, A. Otsuka, H. Yamochi, A. Ono, M. Itoh, M. Yoshida, M. Takigawa, Y. Yoshida, and G. Saito, Pressure-Tuned Exchange Coupling of a Quantum Spin Liquid in the Molecular Triangular Lattice $\kappa-(\mathrm{ET})_{2} \mathrm{Ag}_{2}(\mathrm{CN})_{3}$, Phys. Rev. Lett. 117, 107203 (2016).

[33] Y. Yoshida, H. Ito, M. Maesato, Y. Shimizu, H. Hayama, T. Hiramatsu, Y. Nakamura, H. Kishida, T. Koretsune, C. Hotta, and G. Saito, Spin-disordered quantum phases in a quasi-onedimensional triangular lattice, Nat. Phys. 11, 679 (2015).

[34] M. Tamura, A. Nakao, and R. Kato, Frustration-induced valence-bond ordering in a new quantum triangular antiferromagnet based on $\left[\mathrm{Pd}(\mathrm{dmit})_{2}\right]$, J. Phys. Soc. Jpn. 75, 093701 (2006).

[35] Y. Shimizu, H. Akimoto, H. Tsujii, A. Tajima, and R. Kato, Mott Transition in a Valence-Bond Solid Insulator With a Triangular Lattice, Phys. Rev. Lett. 99, 256403 (2007).

[36] Y. Kurosaki, Y. Shimizu, K. Miyagawa, K. Kanoda, and G. Saito, Mott Transition from a Spin Liquid to a Fermi Liquid in the Spin-Frustrated Organic Conductor $\kappa-(\mathrm{ET})_{2} \mathrm{Cu}_{2}(\mathrm{CN})_{3}$, Phys. Rev. Lett. 95, 177001 (2005).

[37] W. Zheng, R. R. P. Singh, R. H. McKenzie, and R. Coldea, Temperature dependence of the magnetic susceptibility for triangular-lattice antiferromagnets with spatially anisotropic exchange constants, Phys. Rev. B 71, 134422 (2005).

[38] K. Mizukoshi, Y. Nakamura, Y. Yoshida, G. Saito, and H. Kishida, Optical evaluation of electronic anisotropy in a triangular lattice system $\kappa$-(BEDT-TTF $)_{2} \mathrm{~B}(\mathrm{CN})_{4}$, J. Phys. Soc. Jpn. 87, 104708 (2018).

[39] A. V. Chubukov, S. Sachdev, and J. Ye, Theory of twodimensional quantum Heisenberg antiferromagnets with a nearly critical ground state, Phys. Rev. B 49, 11919 (1994).

[40] J. Larsen and C. Lenoir, Synthesis of bis(ethylenedithio)tetrathiafulvalene (BEDT-TTF), Synthesis 1989, 134 (1989).

[41] A. Abragam, The Principles of Nuclear Magnetism (Clarendon, Oxford, 1983).

[42] Y. Shimizu, K. Miyagawa, K. Kanoda, M. Maesato, and G. Saito, Emergence of inhomogeneous moments from spin liquid in the triangular-lattice Mott insulator $\kappa-(\mathrm{ET})_{2} \mathrm{Cu}_{2}(\mathrm{CN})_{3}$, Phys. Rev. B 73, 140407(R) (2006).

[43] D. F. Smith, S. M. De Soto, C. P. Slichter, J. A. Schlueter, A. M. Kini, and R. G. Daugherty, Dzialoshinskii-Moriya interaction in the organic superconductor $\kappa$-(BEDT-TTF $)_{2} \mathrm{Cu}\left[\mathrm{N}(\mathrm{CN})_{2}\right] \mathrm{Cl}$, Phys. Rev. B 68, 024512 (2003).

[44] T. Mizusaki and M. Imada, Gapless quantum spin liquid, stripe, and antiferromagnetic phases in frustrated Hubbard models in two dimensions, Phys. Rev. B 74, 014421 (2006).

[45] E. Ghorbani, L. F. Tocchio, and F. Becca, Variational wave functions for the $S=\frac{1}{2}$ Heisenberg model on the anisotropic triangular lattice: Spin liquids and spiral orders, Phys. Rev. B 93, 085111 (2016).

[46] M. Takigawa, N. Motoyama, H. Eisaki, and S. Uchida, Dynamics in the $S=1 / 2$ One-Dimensional Antiferromagnet $\mathrm{Sr}_{2} \mathrm{CuO}_{3}$ Via ${ }^{63} \mathrm{Cu}$ NMR, Phys. Rev. Lett. 76, 4612 (1996).

[47] S. E. Brown, W. G. Clark, F. Zamborszky, B. J. Klemme, G. Kriza, B. Alavi, C. Merlic, P. Kuhns, and W. Moulton, ${ }^{13}$ C NMR Measurements of the High-Magnetic-Field, Low-Temperature Phases of (TMTTF) ${ }_{2} \mathrm{PF}_{6}$, Phys. Rev. Lett. 80, 5429 (1998). 
[48] M. C. Cross, Effect of magnetic fields on a spin-Peierls transition, Phys. Rev. B 20, 4606 (1979).

[49] S. Inagaki and H. Fukuyama, Spin-Peierls state vs antiferromagnetic state. III. effects of magnetic field, J. Phys. Soc. Jpn. 53, 4386 (1984).

[50] M. Hase, I. Terasaki, K. Uchinokura, M. Tokunaga, N. Miura, and $\mathrm{H}$. Obara, Magnetic phase diagram of the spin-Peierls cuprate $\mathrm{CuGeO}_{3}$, Phys. Rev. B 48, 9616 (1993).

[51] S. E. Brown, W. G. Clark, B. Alavi, D. Hall, M. J. Naughton, D. J. Tantillo, and C. A. Merlic, High-field magnetization of the
spin-Peierls compound (TMTTF) ${ }_{2} \mathrm{PF}_{6}$, Phys. Rev. B 60, 6270 (1999).

[52] R. Kaneko, S. Morita, and M. Imada, Gapless spin-liquid phase in an extended spin 1/2 triangular heisenberg model, J. Phys. Soc. Jpn. 83, 093707 (2014).

[53] H. Ito, T. Asai, Y. Shimizu, H. Hayama, Y. Yoshida, and G. Saito, Pressure-induced superconductivity in the antiferromagnet $\kappa$-(ET) $)_{2} \mathrm{CF}_{3} \mathrm{SO}_{3}$ with quasi-one-dimensional triangular spin lattice, Phys. Rev. B 94, 020503(R) (2016). 